\title{
Cooking, storage, and reheating effect on the formation of cholesterol oxidation products in processed meat products
}

\author{
Muhammad I. Khan ${ }^{1,2}$, Joong-Seok Min ${ }^{3}$, Sang-Ok Lee ${ }^{1}$, Dong Gyun Yim ${ }^{4}$, Kuk-Hwan Seol ${ }^{5}$, Mooha Lee ${ }^{1,6}$
} and Cheorun $\mathrm{JO}^{1 *}$ (I)

\begin{abstract}
Background: Cholesterol is an important biological compound; however, its oxidation products have been proven to be harmful to human health. Cooking, storage, and reheating methods significantly affect the safety of meat products, as they contribute to the production of cholesterol oxidation products (COPs).

Methods: Three cooking methods were used to cook sausages, loin ham, bacon, luncheon meat, and pressed ham, in order to investigate the effect of cooking, storage, and reheating on total cholesterol and on the formation of COPs. Cooked samples were stored at $4{ }^{\circ} \mathrm{C}$ and reheated after 3 and 6 storage days by the same cooking method or by microwaving. The samples were assessed for total lipids, cholesterol, and cholesterol oxides.

Results: The average cholesterol content in the processed meat varied from $76.0 \mathrm{mg} / 100 \mathrm{~g}$ to $201.70 \mathrm{mg} / 100 \mathrm{~g}$. Microwaved ham showed the lowest cholesterol content compared to that of other processed meat products. Significant differences were found in cholesterol content and cholesterol oxidation products depending on cooking, storage, and reheating methods. Six cholesterol oxides were found in processed meat, of which $7 \beta$-hydroxycholesterol and a-epoxides were detected as the major oxidation products.
\end{abstract}

Conclusions: Microwaving and oven grilling resulted in higher production of COPs in processed meat as compared with other cooking methods. Refrigerated storage tended to significantly increase the COPs content.

Keywords: Processed meat products, Cooking and reheating methods, Total cholesterol, Cholesterol oxidation products (COPs)

\section{Background}

During the last two decades, the relationship between diet and health has been widely studied and consumers were encouraged to improve their dietary habits. However, the intake of fats, namely saturated fats, is still higher than that specified by the American Heart Association recommendations, according to which total fats should not exceed $30 \%$ of the total caloric intake and saturated fats should represent less than $10 \%$ of the total calories. Processed meat products are highly appreciated by a large number of populations; however, they are rich in cholesterol, lipids,

\footnotetext{
* Correspondence: cheorun@snu.ac.kr

'Department of Agricultural Biotechnology, Center for Food and Bioconvergence, and Research Institute of Agriculture and Life Science, Seoul National University, Seoul 151-921, South Korea

Full list of author information is available at the end of the article
}

and saturated fatty acids. In general, fats of animal origin are not considered healthy because of their high content of saturated fatty acids and cholesterol [1]. Thermal processing of meat and meat products leads to undesirable changes such as lipid oxidation and protein degradation [2]. Excessive oxidation of meat lipids produces potential precursors of highly reactive aldehydes in tissues and foods, becoming a source of oxidative stress $[3,4]$. These aldehydes can be major contributing factors in several pathological conditions such as atherosclerosis, inflammation, arthritis, Alzheimer's, and Parkinson's disease [5]. Healthy human plasma contains $12.6 \mathrm{mg} / \mathrm{L}$ of COPs [6] and ingestion of foods containing COPs increases these levels in plasma and leads to deleterious health effects.

Cholesterol is a compound of biological importance, widely distributed in food of animal origin. Cholesterol 
contents of meat and meat products varied considerably but general it is less than $70 \mathrm{mg} / 100 \mathrm{~g}$ except for edible offal and it is assumed that one-third of daily intake come from meat and meat products [7]. However, cholesterol oxidation products (COPs) have been proven to be cytotoxic, mutagenic, and carcinogenic [8], and are also considered to be a primary factor responsible for triggering atherosclerosis [9]. COPs are formed when animal-derived foods are subjected to heating and cooking [10], dehydration [11] storage [1], and irradiation [12]. Colorimetric, chromatographic and enzymatic assessment methods are used for assessing cholesterol and its products [13]. Dominguez et al. [14] reported that, among different cooking methods, i.e., roasting, grilling, microwaving, and frying, used for cooking foal meat, microwaved samples showed the highest oxidation products. Lee et al. [12] studied the effects of various cooking and reheating methods on the total cholesterol and on the formation of COPs in beef loin, and observed a significant reduction in cholesterol and an increase in COPs. Hu et al. [15] compared the effects of cooking methods on pork lipid digestibility and on the formation of COPs, and detected a significantly higher COP formation in microwave-treated samples. In a recent study, Freitas et al. [16] reported the reduction in cholesterol content and the increase in COPs, especially 7ketocholesterol, in fish fillets cooked by different methods.

Hence, this study aimed to determine the effects of cooking methods, storage, and reheating on the amount of total lipids and cholesterol, and on the formation of COPs in processed meat products.

\section{Methods}

\section{Reagents and solutions}

Cholesterol, linoleic acid, oleic acid, cholesterol oxide standards [7-ketocholesterol (7-keto), 6-ketocholesterol (6-keto), $7 \alpha$-hydroxycholesterol $(7 \alpha-\mathrm{OH}), 7 \beta$-hydroxy cholesterol $(7 \beta-\mathrm{OH}), 5,6 \alpha$-epoxycholesterol $(5,6 \alpha-\mathrm{EP})$,

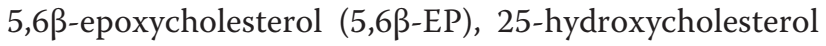
(25-OH), 20-hydroxycholesterol (20-OH), and cholestanetriol (triol)], butylated hydroxytoluene (BHT), Xpyridine, and silicic acid (100 mesh) were purchased from Sigma-Aldrich Co., LLC (Seoul, Korea). Bis(trimethylsilyl)trifluoroacetamide (BSTFA) + $1 \%$ trimethylchlorosilane (TMCS) was obtained from Supelco (Bellefonte, PA, USA). HPLC-grade hexane, ethyl acetate, acetone, methanol, chloroform, Celite 545, and calcium phosphate $\left(\mathrm{CaHPO}_{4} 2 \mathrm{H}_{2} \mathrm{O}\right)$ were purchased from Fisher Scientific Co. (Malvern, PA, USA).

\section{Sample preparation}

Five processed meat products (frankfurter sausage, loin ham, bacon, luncheon meat, and pressed ham (Korean-style luncheon pork)) were purchased from a local market. Frankfurter sausage and bacon were used as such, whereas loin ham, luncheon meat, and pressed ham were cut into $1 \mathrm{~cm}$ thickness. Each product was divided into four groups: fresh (control), pan roasting $(\mathrm{P})$, oven grilling $(\mathrm{O})$, and microwave heating $(\mathrm{M})$. The fresh samples were analyzed immediately after cooking, whereas the cooked samples were stored at refrigerated temperature $\left(4{ }^{\circ} \mathrm{C}\right)$ and reheated after 3 and 6 days using the same cooking method or microwave heating, to simulate restaurant preparation. The treatment groups were the following: pan roasting-pan roasting $(\mathrm{PP})$, pan roasting-microwave heating $(\mathrm{PM})$, oven grilling-oven grilling $(\mathrm{OO})$, oven grilling-microwave heating $(\mathrm{OM})$, and microwave heating-microwave heating (MM). Samples for pan roasting $(\mathrm{P})$ were roasted on an electric grill pan (Excel 10 Electric Grill, Tefal Sa, France) at $180{ }^{\circ} \mathrm{C}$ until the internal temperature of the samples reached $70{ }^{\circ} \mathrm{C}$. After 3 and 6 days of storage, reheating by pan roasting was done at $180{ }^{\circ} \mathrm{C}$ for $5 \mathrm{~min}$ (PP) whereas microwave heating was performed in a microwave oven (M-M270TC, LG Electronics, Korea) with $700 \mathrm{~W}$ power for $2 \mathrm{~min}(\mathrm{PM})$. The samples were cooked by oven grilling in a convection oven (GR-643HT, Tong Yang Magic, Korea) at $150{ }^{\circ} \mathrm{C}$ until the internal temperature reached $70{ }^{\circ} \mathrm{C}$. After 3 and 6 days of storage, reheating by oven grilling was done at $150{ }^{\circ} \mathrm{C}$ for $10 \mathrm{~min}(\mathrm{OO})$ using the same oven, whereas microwave heating was performed in a microwave oven with $700 \mathrm{~W}$ power for $2 \mathrm{~min}(\mathrm{OM})$. For the MM group, the samples were cooked in a microwave oven for $10 \mathrm{~min}$ and reheated in a microwave oven for 2 min, after 3 and 6 days of storage.

\section{Determination of total cholesterol}

The fat $(0.5 \mathrm{~g})$ was diluted in $10 \mathrm{~mL}$ of freshly prepared methanolic potassium hydroxide solution $(1 \mathrm{M})$ and $1 \mathrm{~g}$ of sea sand was added. The mixture was heated for $25 \mathrm{~min}$ and the supernatant was transferred with a pipette into a $25 \mathrm{~mL}$ volumetric flask. The residue was boiled with $6 \mathrm{~mL}$ of isopropanol under reflux condenser for $5 \mathrm{~min}$, and the solution was collected, cooled, and diluted to the mark with isopropanol. The turbid solutions were filtered through a Whatman No. 1 filter paper (Whatman Inc., Clifton, NJ). The clear aliquot was used for the cholesterol assay following the kit instructions (Cat. No 139050, Boehringer Mannheim, Germany). The blank sample was prepared by mixing $0.4 \mathrm{~mL}$ of the extracted sample solution and $5 \mathrm{~mL}$ of solution 4 (cholesterol reagent mixture). The sample solution was obtained by mixing $2.5 \mathrm{~mL}$ of the extracted sample solution and $0.02 \mathrm{~mL}$ of solution 3 . The prepared blank and sample solutions were sealed with paraffin film and incubated at $37-40{ }^{\circ} \mathrm{C}$ for $60 \mathrm{~min}$. The absorbance values of the blank (A1) and of the sample (A2) were determined using an UV spectrophotometer (UV1601, Shimadzu Co., Japan) at $405 \mathrm{~nm}$. Cholesterol contents $(\mathrm{mg} / 100 \mathrm{~g})$ were calculated using the following equation: 


$$
\begin{aligned}
\text { Cholesterol content }(\mathrm{mg} / 100 \mathrm{~g}) \\
= \\
\quad[0.711 \times(\mathrm{A} 2-\mathrm{A} 1) / \text { sample weight }(\mathrm{g})] \times 100 \\
\quad \times 25
\end{aligned}
$$

\section{Cholesterol Oxidation Products (COPs)}

Cholesterol oxidation products were analyzed according to the method of Lee et al. [17]. For the separation of COPs, a solid-phase column was prepared $[18,19]$ by mixing silicic acid, Celite 545, and $\mathrm{CaHPO}_{4} 2 \mathrm{H}_{2} \mathrm{O}$ (10:9:1, wt/wt/wt) with $30 \mathrm{~mL}$ chloroform, and the mixture was packed in a glass column $(12 \mathrm{~mm} \times 30 \mathrm{~cm})$. The prepared column was repeatedly prewashed with $5 \mathrm{~mL}$ of hexane before sample application. The total lipids were extracted following the method of Folch et al. [20]. The lipid sample $(0.2 \mathrm{~g})$ was dissolved in $2 \mathrm{~mL}$ hexane:ethyl acetate $(100: 2, \mathrm{v} / \mathrm{v})$ and applied to a prewashed column. The sample container was washed twice with $2 \mathrm{~mL}$ hexane:ethyl acetate and the wash solvent was applied to the column. Neutral lipid and cholesterol (phospholipids) were removed by adding $50 \mathrm{~mL}$ of solvent I $\left(\mathrm{CHCl}_{3}: \mathrm{CH}_{3} \mathrm{OH}=2: 1, \mathrm{v} / \mathrm{v}\right)$ and $60 \mathrm{~mL}$ of solvent II (hexane:ethyl acetate $=4: 1, \mathrm{v} / \mathrm{v}$ ). Solvent III (40 mL, acetone:ethyl acetate:methanol $=10: 10: 1, \quad \mathrm{v} / \mathrm{v} / \mathrm{v}$ ) was used at a $1 \mathrm{~mL} / \mathrm{min}$ flow rate to elute the COPs. The collected solutions were dried on a $50{ }^{\circ} \mathrm{C}$ hot plate with nitrogen gas flushing. The dried extracts were derivatized by heating at $80{ }^{\circ} \mathrm{C}$ for $1 \mathrm{~h}$ in the presence of $200 \mu \mathrm{L}$ pyridine and $100 \mu \mathrm{L}$ sylon-bis(trimethylsilyl)trifluoroacetamide $+1 \%$ trimethylchlorosilane. The COPs were analyzed using a gas chromatograph (HP 5890 plus) equipped with an oncolumn capillary injector and a flame ionization detector. Identification of cholesterol and COPs was based on the comparison of the retention times of the samples with those of the standards, on co-chromatography, and on the characteristics of the absorption spectra.

\section{Statistical analysis}

Statistical analysis was performed by one-way analysis of variation (ANOVA) using SAS software (SAS, Release 8.01, SAS Institute Inc., Cary, NC), and Duncan's multiple range test was employed to differentiate the significance among mean values.

\section{Results and discussion}

\section{Total fat and cholesterol content}

Total fat and cholesterol contents of processed meat products cooked and reheated by different methods after 3 and 6 days of storage at $4{ }^{\circ} \mathrm{C}$ are shown in Tables 1 and 2 . The highest fat content was found in fresh bacon (48.5\%), followed by sausages $(24.7 \%)$, luncheon meats (23.6 \%), and pressed ham (16.3\%), and the lowest fat was found in fresh loin meat $(8.3 \%)$. The fat content of meat products varied significantly as a result of cooking and reheating as well as during storage. Bacon showed non-significant changes upon cooking and reheating; however, a considerable reduction in fat content was observed after storing the product for 6 days. The cholesterol content of fresh samples did not vary significantly during storage at $4{ }^{\circ} \mathrm{C}$ for 6 days (Table 2). Cooking and reheating of meat products greatly affect their cholesterol content, and maximum reduction in cholesterol was observed for oven grilling and microwave cooking

Table 1 Changes of the crude fat content (\%) of processed

\begin{tabular}{|c|c|c|c|c|}
\hline \multirow[t]{2}{*}{ Sample } & \multirow{2}{*}{$\begin{array}{l}\text { Cooking } \\
\text { method }\end{array}$} & \multicolumn{3}{|c|}{ Re-heating (day) } \\
\hline & & 0 & 3 & 6 \\
\hline \multirow[t]{6}{*}{ Sausage } & Fresh & $24.7 \pm 0.09^{\mathrm{AB}}$ & $24.5 \pm 0.66^{\mathrm{AB}}$ & $24.1 \pm 0.53^{A}$ \\
\hline & PP & $24.6 \pm 0.0^{\mathrm{Ba}}$ & $23.6 \pm 0.01^{\mathrm{BCb}}$ & $23.5 \pm 0.10^{\mathrm{Bb}}$ \\
\hline & PM & $24.6 \pm 0.0^{\mathrm{Ba}}$ & $23.0 \pm 0.98^{\mathrm{Cb}}$ & $22.8 \pm 0.13^{\mathrm{cb}}$ \\
\hline & $\mathrm{OO}$ & $25.2 \pm 0.78^{\mathrm{Aa}}$ & $24.7 \pm 0.50^{\mathrm{Aa}}$ & $23.8 \pm 0.01^{\mathrm{ABb}}$ \\
\hline & $\mathrm{OM}$ & $25.2 \pm 0.78^{\mathrm{Aa}}$ & $24.0 \pm 0.11^{\mathrm{ABCb}}$ & $23.7 \pm 0.10^{\mathrm{ABb}}$ \\
\hline & MM & $23.3 \pm 0.06^{\mathrm{Ca}}$ & $23.0 \pm 0.11^{\mathrm{cb}}$ & $23.0 \pm 0.12^{\mathrm{Cb}}$ \\
\hline \multirow[t]{6}{*}{ Loin ham } & Fresh & $8.3 \pm 0.09^{A}$ & $8.1 \pm 0.52^{\mathrm{A}}$ & $8.1 \pm 0.56^{A}$ \\
\hline & PP & $5.7 \pm 0.01^{\mathrm{Ba}}$ & $5.5 \pm 0.01^{\mathrm{Bb}}$ & $5.3 \pm 0.13^{B C}$ \\
\hline & PM & $5.7 \pm 0.01^{\mathrm{Ba}}$ & $5.4 \pm 0.01^{\mathrm{Bb}}$ & $4.8 \pm 0.19^{B C}$ \\
\hline & $\mathrm{OO}$ & $6.8 \pm 0.01^{\mathrm{Ba}}$ & $5.8 \pm 0.03^{\mathrm{Bb}}$ & $4.9 \pm 0.04^{B C}$ \\
\hline & OM & $6.8 \pm 0.01^{\mathrm{Ba}}$ & $5.4 \pm 0.10^{\mathrm{Bb}}$ & $5.0 \pm 012^{B C}$ \\
\hline & MM & $5.5 \pm 0.01^{\mathrm{Bb}}$ & $5.8 \pm 0.11^{\mathrm{Ba}}$ & $5.1 \pm 0.08^{B C}$ \\
\hline \multirow[t]{6}{*}{ Bacon } & Fresh & $48.5 \pm 0.51$ & $48.7 \pm 0.84$ & $48.6 \pm 1.00$ \\
\hline & PP & $49.0 \pm 0.57^{a}$ & $47.7 \pm 0.01^{b}$ & $46.6 \pm 0.09^{c}$ \\
\hline & PM & $49.0 \pm 0.57^{\mathrm{a}}$ & $47.5 \pm 0.71^{b}$ & $44.6 \pm 0.56^{c}$ \\
\hline & $\mathrm{OO}$ & $47.0 \pm 0.0^{\mathrm{a}}$ & $45.9 \pm 0.29^{b}$ & $45.7 \pm 0.09^{b}$ \\
\hline & $\mathrm{OM}$ & $47.0 \pm 0.01^{b}$ & $48.6 \pm 0.38^{a}$ & $43.8 \pm 0.10^{c}$ \\
\hline & MM & $48.8 \pm 0.62^{a}$ & $45.8 \pm 0.02^{b}$ & $42.9 \pm 0.10^{c}$ \\
\hline \multirow{6}{*}{$\begin{array}{l}\text { Luncheon } \\
\text { meat }\end{array}$} & Fresh & $23.6 \pm 1.10^{\mathrm{AB}}$ & $23.2 \pm 0.71^{A}$ & $23.2 \pm 0.69^{B C}$ \\
\hline & PP & $24.0 \pm 0.07^{\mathrm{Ab}}$ & $27.1 \pm 0.11^{\mathrm{Aa}}$ & $27.2 \pm 0.14^{\mathrm{Aa}}$ \\
\hline & PM & $24.0 \pm 0.07^{\mathrm{Ab}}$ & $23.6 \pm 0.08^{A c}$ & $25.9 \pm 0.08^{\mathrm{Aba}}$ \\
\hline & $\mathrm{O}$ & $24.0 \pm 0.11^{A C}$ & $26.8 \pm 0.10^{\mathrm{Aa}}$ & $24.6 \pm 0.21^{\mathrm{ABb}}$ \\
\hline & OM & $24.0 \pm 0.11^{\mathrm{Ba}}$ & $20.3 \pm 0.10^{\mathrm{Bb}}$ & $19.2 \pm 0.10^{c c}$ \\
\hline & MM & $23.4 \pm 0.09^{\mathrm{AB}}$ & $24.3 \pm 0.08^{\mathrm{A}}$ & $23.5 \pm 0.12^{\mathrm{ABC}}$ \\
\hline \multirow[t]{6}{*}{ Press Ham } & Fresh & $16.3 \pm 0.10^{B}$ & $16.4 \pm 0.31^{B}$ & $16.2 \pm 0.26^{\mathrm{B}}$ \\
\hline & PP & $18.2 \pm 0.09^{A C}$ & $21.4 \pm 0.01^{\mathrm{Aa}}$ & $19.6 \pm 0.08^{\mathrm{Ab}}$ \\
\hline & PM & $18.2 \pm 0.09^{A C}$ & $18.8 \pm 0.10^{\mathrm{Ab}}$ & $19.8 \pm 0.13^{\mathrm{Aa}}$ \\
\hline & $\mathrm{OO}$ & $20.1 \pm 0.08^{\mathrm{Aa}}$ & $18.4 \pm 0.16^{\mathrm{Ab}}$ & $19.9 \pm 0.55^{\mathrm{Aa}}$ \\
\hline & $\mathrm{OM}$ & $20.1 \pm 0.08^{\mathrm{Ab}}$ & $16.6 \pm 0.07^{\mathrm{Ac}}$ & $21.2 \pm 0.01^{\mathrm{Aa}}$ \\
\hline & MM & $20.3 \pm 0.07^{A C}$ & $21.4 \pm 0.12^{\mathrm{Aa}}$ & $20.6 \pm 0.08^{A b}$ \\
\hline
\end{tabular}
meat products in fresh, cooked, and re-heated at day 3 and 6 of storage

Means \pm SE with different superscript in the same row $\left(^{\mathrm{a}-\mathrm{C}}\right)$ and column $\left({ }^{\mathrm{A}-\mathrm{C}}\right)$ differ significantly $(p<0.05)$

Pan roasting (cooking) + pan roasting (re-heating), PP; pan roasting (cooking) + microwaving (re-heating), PM; oven grilling (cooking) + oven grilling (re-heating), OO; oven grilling (cooking) + microwaving (re-heating), OM; microwaving (cooking) + microwaving (re-heating), MM 
Table 2 Changes of cholesterol content ( $\mathrm{mg} / 100 \mathrm{~g}$ ) of meat products in fresh, cooked, and re-heated at day 3 and 6 of storage

\begin{tabular}{|c|c|c|c|c|}
\hline \multirow[t]{2}{*}{ Sample } & \multirow{2}{*}{$\begin{array}{l}\text { Cooking } \\
\text { method }\end{array}$} & \multicolumn{3}{|l|}{ Re-heating (day) } \\
\hline & & 0 & 3 & 6 \\
\hline \multirow[t]{6}{*}{ Sausage } & Fresh & $96.2 \pm 11.52$ & $95.3 \pm 11.24$ & $94.7 \pm 10.99^{C}$ \\
\hline & PP & $103.3 \pm 6.57^{b}$ & $86.0 \pm 4.63^{c}$ & $137.9 \pm 4.78^{\mathrm{Aa}}$ \\
\hline & PM & $103.3 \pm 6.57^{b}$ & $84.6 \pm 5.11^{c}$ & $119.5 \pm 3.64^{\mathrm{Ba}}$ \\
\hline & $\mathrm{OO}$ & $93.0 \pm 4.22$ & $88.3 \pm 11.88$ & $91.5 \pm 1.14^{C}$ \\
\hline & OM & $93.0 \pm 4.22^{\mathrm{a}}$ & $74.3 \pm 6.25^{b}$ & $93.0 \pm 3.02^{\mathrm{Ca}}$ \\
\hline & MM & $93.4 \pm 7.07$ & $90.1 \pm 4.07$ & $91.2 \pm 4.05^{C}$ \\
\hline \multirow[t]{6}{*}{ Loin ham } & Fresh & $91.7 \pm 7.93^{B}$ & $91.9 \pm 7.41^{\mathrm{A}}$ & $91.9 \pm 7.65^{\mathrm{A}}$ \\
\hline & PP & $54.6 \pm 1.61^{\mathrm{cb}}$ & $47.8 \pm 3.20^{\mathrm{Cc}}$ & $89.8 \pm 2.82^{\mathrm{Aa}}$ \\
\hline & PM & $54.6 \pm 1.61^{\mathrm{Cc}}$ & $71.8 \pm 2.54^{\mathrm{Ba}}$ & $64.3 \pm 3.79^{\mathrm{cb}}$ \\
\hline & $\mathrm{OO}$ & $101.4 \pm 6.03^{\mathrm{Aa}}$ & $52.5 \pm 1.54^{\mathrm{Cc}}$ & $85.6 \pm 2.86^{\mathrm{ABb}}$ \\
\hline & OM & $101.4 \pm 6.03^{\mathrm{Aa}}$ & $55.6 \pm 3.04^{\mathrm{Cc}}$ & $79.8 \pm 0.91^{\mathrm{Bb}}$ \\
\hline & MM & $45.1 \pm 1.83^{\mathrm{Da}}$ & $49.4 \pm 4.62^{\mathrm{Ca}}$ & $29.5 \pm 0.37^{\mathrm{Db}}$ \\
\hline \multirow[t]{6}{*}{ Bacon } & Fresh & $201.70 \pm 7.95^{\mathrm{A}}$ & $201.2 \pm 8.68^{B C}$ & $200.8 \pm 7.56^{B}$ \\
\hline & PP & $155.4 \pm 8.95^{B C}$ & $205.7 \pm 7.61^{\mathrm{ABb}}$ & $238.0 \pm 9.32^{\mathrm{Aa}}$ \\
\hline & PM & $155.4 \pm 8.95^{\mathrm{Bb}}$ & $232.4 \pm 18.58^{\mathrm{Aa}}$ & $218.1 \pm 18.81^{\mathrm{Aba}}$ \\
\hline & $\mathrm{OO}$ & $140.8 \pm 23.0^{\mathrm{Bb}}$ & $158.8 \pm 27.23^{\mathrm{Dab}}$ & $198.3 \pm 9.19^{\mathrm{Ba}}$ \\
\hline & OM & $140.8 \pm 23.0^{B}$ & $173.9 \pm 18.08^{C D}$ & $168.1 \pm 10.21^{C}$ \\
\hline & MM & $151.6 \pm 4.84^{B}$ & $174.0 \pm 6.48^{C D}$ & $163.5 \pm 12.63^{C}$ \\
\hline \multirow[t]{6}{*}{ Luncheon meat } & Fresh & $106.2 \pm 5.06^{A}$ & $106.1 \pm 4.16^{A}$ & $106.2 \pm 4.83^{B C}$ \\
\hline & PP & $92.8 \pm 9.80^{B}$ & $94.2 \pm 14.45^{\mathrm{AB}}$ & $81.8 \pm 5.29^{C}$ \\
\hline & PM & $92.8 \pm 9.80^{B}$ & $98.3 \pm 18.34^{\mathrm{AB}}$ & $125.3 \pm 15.61^{\mathrm{B}}$ \\
\hline & $\mathrm{OO}$ & $91.8 \pm 2.15^{\mathrm{Ba}}$ & $77.1 \pm 3.94^{\mathrm{Bb}}$ & $80.1 \pm 2.27^{\mathrm{Ca}}$ \\
\hline & $\mathrm{OM}$ & $91.8 \pm 2.15^{B}$ & $91.7 \pm 6.07^{\mathrm{AB}}$ & $121.8 \pm 31.64^{B}$ \\
\hline & MM & $79.7 \pm 6.93^{B C}$ & $111.1 \pm 13.55^{\mathrm{Ab}}$ & $214.4 \pm 6.50^{\mathrm{Aa}}$ \\
\hline \multirow[t]{6}{*}{ Press ham } & Fresh & $76.0 \pm 7.30$ & $75.5 \pm 6.85^{B}$ & $75.4 \pm 6.61^{B}$ \\
\hline & PP & $81.0 \pm 4.38^{\mathrm{a}}$ & $70.6 \pm 3.84^{\mathrm{Bb}}$ & $53.2 \pm 4.35^{\mathrm{BCC}}$ \\
\hline & PM & $81.0 \pm 4.38$ & $92.3 \pm 11.04^{\mathrm{A}}$ & $52.7 \pm 43.23^{B C}$ \\
\hline & $\mathrm{OO}$ & $67.9 \pm 9.10$ & $65.4 \pm 9.43^{B}$ & $69.6 \pm 3.99^{B}$ \\
\hline & $\mathrm{OM}$ & $67.9 \pm 9.10^{\mathrm{a}}$ & $59.4 \pm 11.87^{\mathrm{Ba}}$ & $31.1 \pm 1.71^{\mathrm{Cb}}$ \\
\hline & MM & $67.8 \pm 12.47^{b}$ & $62.2 \pm 6.01^{\mathrm{Bb}}$ & $191.5 \pm 9.40^{\mathrm{Aa}}$ \\
\hline
\end{tabular}

Means \pm SE with different superscript in the same row $\left(^{\mathrm{a}-\mathrm{C}}\right)$ and column $\left({ }^{\mathrm{A}-\mathrm{C}}\right)$ differ significantly $(p<0.05)$

Pan roasting (cooking) + pan roasting (re-heating), PP; pan roasting (cooking) + microwaving (re-heating), PM; oven grilling (cooking) + oven grilling (re-heating), OO; oven grilling (cooking) + microwaving (re-heating), OM; microwaving (cooking) + microwaving (re-heating), MM

and reheating. Among the tested samples, the highest cholesterol content $(201.70 \mathrm{mg} / 100 \mathrm{~g})$ was observed in bacon, and the lowest $(76.00 \mathrm{mg} / 100 \mathrm{~g})$ in pressed ham. In the present study, the wide range of cholesterol content from pressed ham to bacon is probably due to a high variability in the original samples. Rodriguez-Estrada et al. [2] reported the presence of higher amounts of cholesterol in fresh samples compared with the cooked samples, and attributed these differences to the loss of cholesterol during the cooking process. Cooking and reheating resulted in the oxidation of cholesterol and production of COPs.
These findings are in agreement with the results obtained by Freitas et al. [16], who reported a decrease in the cholesterol content of fish fillets and an increase in COP formation upon cooking. Badiani et al. [21] reported that cooking induced a remarkable decrease in moisture content, which varies depending on the different cooking methods, leading to various cholesterol levels due to the differences in cooking and reheating techniques. The results obtained in the current study also confirm the findings of Baggio and Bragagnolo [22], who observed that storage time did not alter the cholesterol content of processed meat samples. 


\section{Cooking methods and COP formation}

The data expressed in Table 3 shows the impact of the cooking methods on the formation of COPs in fresh meat products. $7 \mathrm{\beta}-\mathrm{OH}$ was observed in sausages and bacon for all cooking methods, whereas only microwave cooking produces $7 \beta-\mathrm{OH}$ in loin ham. However, $7 \beta-\mathrm{OH}$ was not detected in luncheon meat and pressed ham after cooking by all methods. The highest level of $7 \beta-\mathrm{OH}(360.54 \mu \mathrm{g} /$ $100 \mathrm{~g}$ ) was observed in sausages cooked by oven grilling. A low level of $25-\mathrm{OH}$ was found in fresh as well as in cooked loin ham and in oven grilled luncheon meat. A high 25-OH level was observed in sausages cooked by microwave. Cholestanetriol was detected in microwaved sausage $(171.6 \mu \mathrm{g} / 100 \mathrm{~g})$, pan-roasted bacon $(184.2 \mu \mathrm{g} / 100 \mathrm{~g})$, and fresh $(153.8 \mu \mathrm{g} / 100 \mathrm{~g})$ and oven grilled luncheon meat $(256.9 \mu \mathrm{g} / 100 \mathrm{~g})$. Low levels of $\alpha$-epoxide were found in fresh, pan roasted, and microwaved sausages, and in oven grilled loin ham and luncheon meat. $20 \alpha-\mathrm{OH}$ and 7-keto were not detected in any of the meat products for all the tested cooking methods.

The ratio between the total amount of COPs and cholesterol in the different meat products cooked by different methods was found to be very low $(0-0.73 \%)$. The highest value was observed in sausages cooked by microwave $(0.73 \%)$, whereas bacon contained the highest cholesterol content (Table 2) and a low total COPs (0.16\%) (Table 3). The presence of a higher amount of $7 \beta-\mathrm{OH}$ in cooked meat products is in agreement with the previous finding of $\mathrm{Du}$ et al. [23], who observed a higher level of $7 \alpha-\mathrm{OH}$ and $7 \beta-\mathrm{OH}$ compared with other COPs in cooked turkey meat. Broncano et al. [24] investigated different cooking methods and their role in lipid oxidation and in the formation of COPs, and reported the presence of higher levels of $7 \alpha-\mathrm{OH}$ and $7 \beta-\mathrm{OH}$ than other COPs. The absence or lower level of triol, $\alpha$-epoxide, $20 \alpha-\mathrm{OH}$, and 7 keto than $7 \beta-\mathrm{OH}$ is in accordance with the findings of Grau et al. [25] and Ghiretti et al. [26]. The higher COP formation owing to microwaving confirms the results of Dominguez et al. [14], who, upon cooking foal meat using roasting, grilling, microwaving, and frying, detected the highest lipid oxidation in microwaved samples. Serra et al. [27] reported 7 $3-\mathrm{OH}$ and 7-keto as the primary products of cholesterol oxidation, and $\alpha$ - and $\beta$-epoxides as the secondary oxidation products.

\section{Storage, reheating, and COP formation}

The cooked meat products stored at $4{ }^{\circ} \mathrm{C}$ and reheated after 3 and 6 storage days were assessed for the formation of COPs (Tables 4 and 5). It is evident from the data presented in Table 4 that the COPs observed in cooked

Table 3 The amount of cholesterol oxidation products $(\mu \mathrm{g} / 100 \mathrm{~g})$ of meat products with different cooking methods

\begin{tabular}{|c|c|c|c|c|c|c|c|c|}
\hline \multirow[t]{2}{*}{ Sample } & \multirow{2}{*}{$\begin{array}{l}\text { Cooking } \\
\text { method }\end{array}$} & \multicolumn{6}{|c|}{ Cholesterol Oxidation Products } & \multirow{2}{*}{$\begin{array}{l}\text { COPs/cholesterol } \\
\text { (\%) }\end{array}$} \\
\hline & & $7 \beta-\mathrm{OH}$ & $20 \mathrm{a}-\mathrm{OH}$ & $25-\mathrm{OH}$ & Triol & a-epoxide & 7-keto & \\
\hline \multirow[t]{4}{*}{ Sausage } & Fresh & n.d. & n.d. & n.d. & n.d. & $20.2 \pm 34.94$ & n.d. & $0.02 \pm 0.04$ \\
\hline & $P$ & $58.3 \pm 100.98$ & n.d. & n.d. & n.d. & $29.9 \pm 51.69$ & n.d. & $0.08 \pm 0.14$ \\
\hline & O & $360.5 \pm 624.48$ & n.d. & n.d. & n.d. & - & n.d. & $0.36 \pm 0.63$ \\
\hline & M & $204.3 \pm 228.85$ & n.d. & $359.8 \pm 598.90$ & $171.6 \pm 297.14$ & $17.4 \pm 30.08$ & n.d. & $0.73 \pm 0.71$ \\
\hline \multirow[t]{4}{*}{ Loin ham } & Fresh & n.d. & n.d. & $43.0 \pm 74.39$ & n.d. & n.d. & n.d. & $0.05 \pm 0.08$ \\
\hline & P & n.d. & n.d. & $5.5 \pm 9.49$ & n.d. & n.d. & n.d. & $0.01 \pm 0.01$ \\
\hline & O & n.d. & n.d. & $22.0 \pm 38.01$ & n.d. & $11.6 \pm 20.12$ & n.d. & $0.04 \pm 0.07$ \\
\hline & M & $24.6 \pm 33.33$ & n.d. & $127.3 \pm 171.25$ & n.d. & n.d. & n.d. & $0.16 \pm 0.21$ \\
\hline \multirow[t]{4}{*}{ Bacon } & Fresh & n.d. & n.d. & n.d. & n.d. & n.d. & n.d. & $0.00 \pm 0.00$ \\
\hline & $P$ & $134.2 \pm 26.97$ & n.d. & n.d. & $184.2 \pm 319.09$ & n.d. & n.d. & $0.16 \pm 0.15$ \\
\hline & $\mathrm{O}$ & $110.6 \pm 191.50$ & n.d. & n.d. & n.d. & n.d. & n.d. & $0.05 \pm 0.09$ \\
\hline & M & $304.4 \pm 527.25$ & n.d. & n.d. & n.d. & n.d. & n.d. & $0.16 \pm 0.27$ \\
\hline \multirow[t]{4}{*}{ Luncheon meat } & Fresh & n.d. & n.d. & n.d. & $153.8 \pm 266.35$ & n.d. & n.d. & $0.15 \pm 0.26$ \\
\hline & $P$ & n.d. & n.d. & n.d. & n.d. & n.d. & n.d. & $0.00 \pm 0.00$ \\
\hline & $\mathrm{O}$ & n.d. & n.d. & $30.9 \pm 53.49$ & $256.9 \pm 445.03$ & $50.7 \pm 87.73$ & n.d. & $0.32 \pm 0.48$ \\
\hline & M & n.d. & n.d. & n.d. & n.d. & n.d. & n.d. & $0.00 \pm 0.00$ \\
\hline \multirow[t]{4}{*}{ Press ham } & Fresh & n.d. & n.d. & n.d. & n.d. & n.d. & n.d. & $0.00 \pm 0.00$ \\
\hline & $P$ & n.d. & n.d. & n.d. & n.d. & n.d. & n.d. & $0.00 \pm 0.00$ \\
\hline & $\mathrm{O}$ & n.d. & n.d. & n.d. & n.d. & n.d. & n.d. & $0.00 \pm 0.00$ \\
\hline & M & n.d. & n.d. & n.d. & n.d. & n.d. & n.d. & $0.00 \pm 0.00$ \\
\hline
\end{tabular}

Pan roasting, $\mathrm{P}$; oven grilling, $\mathrm{O}$; microwaving, $\mathrm{M}$; $7 \beta$-hydroxycholesterol, $7 \beta-\mathrm{OH}$; 20a-hydroxycholesterol, 20a-OH; 25-hydroxycholesterol, 25-OH; cholestane-3 $3,5 a, 6 \beta$-triol, triol; cholesterol-5a,6a-epoxide, a-epoxide; 7-ketocholesterol, 7-keto 
Table 4 The amount of cholesterol oxidation products $(\mu \mathrm{g} / 100 \mathrm{~g})$ of meat products cooked with different methods and re-heated by microwaving at day 3 of storage at $4{ }^{\circ} \mathrm{C}$

\begin{tabular}{|c|c|c|c|c|c|c|c|c|}
\hline \multirow[t]{2}{*}{ Sample } & \multirow{2}{*}{$\begin{array}{l}\text { Cooking } \\
\text { method }\end{array}$} & \multicolumn{6}{|c|}{ Cholesterol Oxidation Products } & \multirow{2}{*}{$\begin{array}{l}\text { COPs/cholestero } \\
(\%)\end{array}$} \\
\hline & & $7 \beta-\mathrm{OH}$ & $20 \mathrm{a}-\mathrm{OH}$ & $25-\mathrm{OH}$ & Triol & a-epoxide & 7-keto & \\
\hline \multirow[t]{6}{*}{ Sausage } & Fresh & $12.7 \pm 14.14$ & n.d. & n.d. & n.d. & n.d. & n.d. & $0.01 \pm 0.01$ \\
\hline & PP & $155.1 \pm 268.60$ & n.d. & n.d. & $283.0 \pm 49.01$ & n.d. & n.d. & $0.42 \pm 0.42$ \\
\hline & PM & $42.6 \pm 73.81$ & n.d. & $396.1 \pm 686.09$ & n.d. & $15.7 \pm 27.24^{B}$ & n.d. & $0.46 \pm 0.69$ \\
\hline & ○ & $300.3 \pm 520.14$ & n.d. & n.d. & n.d. & n.d. & n.d. & $0.34 \pm 0.59$ \\
\hline & OM & $147.3 \pm 140.60$ & n.d. & $278.2 \pm 481.78$ & $87.8 \pm 152.00$ & n.d. & n.d. & $0.57 \pm 0.49$ \\
\hline & MM & $55.2 \pm 95.63$ & n.d. & $381.2 \pm 660.22$ & n.d. & $315.0 \pm 320.37^{B}$ & n.d. & $0.86 \pm 1.07$ \\
\hline \multirow[t]{6}{*}{ Loin ham } & Fresh & n.d. & n.d. & $43.5 \pm 75.38$ & n.d. & $26.0 \pm 45.07^{B}$ & n.d. & $0.07 \pm 0.13$ \\
\hline & PP & $22.9 \pm 29.78$ & n.d. & $196.5 \pm 153.41$ & n.d. & $125.2 \pm 216.91^{B}$ & n.d. & $0.64 \pm 0.63$ \\
\hline & PM & $9.9 \pm 17.08$ & n.d. & $13.5 \pm 23.38$ & $26.4 \pm 45.70$ & $19.4 \pm 23.69^{B}$ & n.d. & $0.13 \pm 0.10$ \\
\hline & ○ & $8.9 \pm 8.05$ & n.d. & $58.7 \pm 101.69$ & $54.6 \pm 94.51$ & $77.1 \pm 124.63^{B}$ & n.d. & $0.20 \pm 0.20$ \\
\hline & $\mathrm{OM}$ & $8.6 \pm 14.85$ & n.d. & $25.0 \pm 43.34$ & $16.4 \pm 28.44$ & $79.7 \pm 138.02^{\mathrm{B}}$ & n.d. & $0.13 \pm 0.16$ \\
\hline & MM & $56.6 \pm 98.02$ & n.d. & $241.6 \pm 213.09$ & n.d. & $34.2 \pm 59.23^{B}$ & n.d. & $0.73 \pm 0.64$ \\
\hline \multirow[t]{6}{*}{ Bacon } & Fresh & n.d. & n.d. & n.d. & n.d. & n.d. & n.d. & $0.00 \pm 0.00$ \\
\hline & PP & $899.1 \pm 1557.30$ & n.d. & n.d. & $245.6 \pm 425.41$ & n.d. & n.d. & $0.71 \pm 0.84$ \\
\hline & PM & $120.7 \pm 209.00$ & n.d. & n.d. & $109.7 \pm 189.93$ & n.d. & n.d. & $0.14 \pm 0.13$ \\
\hline & ○० & $94.7 \pm 164.09$ & n.d. & $247.1 \pm 427.91$ & n.d. & $751.2 \pm 909.52^{A}$ & n.d. & $0.81 \pm 0.95$ \\
\hline & $\mathrm{OM}$ & $111.6 \pm 193.21$ & n.d. & n.d. & $212.5 \pm 368.04$ & n.d. & n.d. & $0.25 \pm 0.24$ \\
\hline & MM & $589.4 \pm 1020.48$ & n.d. & $667.2 \pm 1155.64$ & n.d. & n.d. & n.d. & $0.84 \pm 0.73$ \\
\hline \multirow[t]{6}{*}{ Luncheon meat } & Fresh & n.d. & n.d. & n.d. & $156.6 \pm 271.45$ & n.d. & n.d. & $0.15 \pm 0.27$ \\
\hline & PP & n.d. & n.d. & n.d. & n.d. & n.d. & n.d. & $0.00 \pm 0.00$ \\
\hline & PM & $150.5 \pm 260.58$ & n.d. & $52.6 \pm 91.14$ & $205.8 \pm 356.42$ & $37.5 \pm 37.68^{B}$ & $26.3 \pm 45.51$ & $0.48 \pm 0.44$ \\
\hline & $\mathrm{OO}$ & n.d. & n.d. & $10.7 \pm 18.51$ & $107.1 \pm 185.42$ & n.d. & n.d. & $0.13 \pm 0.22$ \\
\hline & $\mathrm{OM}$ & $87.7 \pm 151.86$ & n.d. & $95.1 \pm 164.77$ & n.d. & n.d. & n.d. & $0.20 \pm 0.17$ \\
\hline & MM & $231.2 \pm 440.37$ & n.d. & n.d. & n.d. & n.d. & n.d. & $0.32 \pm 0.55$ \\
\hline \multirow[t]{6}{*}{ Press ham } & Fresh & n.d. & n.d. & $48.1 \pm 76.50$ & n.d. & n.d. & n.d. & $0.07 \pm 0.11$ \\
\hline & PP & n.d. & n.d. & n.d. & $168.4 \pm 162.53$ & n.d. & n.d. & $0.22 \pm 0.21$ \\
\hline & PM & n.d. & n.d. & $112.2 \pm 194.33$ & n.d. & n.d. & n.d. & $0.15 \pm 0.26$ \\
\hline & ০० & $497.0 \pm 860.89$ & n.d. & n.d. & n.d. & n.d. & n.d. & $0.63 \pm 1.10$ \\
\hline & OM & n.d. & n.d. & $91.8 \pm 159.05$ & n.d. & n.d. & n.d. & $0.15 \pm 0.26$ \\
\hline & MM & n.d. & n.d. & $106.5 \pm 184.50$ & n.d. & n.d. & n.d. & $0.13 \pm 0.22$ \\
\hline
\end{tabular}

Means \pm SE with different superscript in the same column $\left({ }^{A-C}\right)$ differ significantly

Pan roasting (cooking) + pan roasting (re-heating), PP; pan roasting (cooking) + microwaving (re-heating), PM; oven grilling (cooking) + oven grilling (re-heating), OO; oven grilling (cooking) + microwaving (re-heating), OM; microwaving (cooking) + microwaving (re-heating), MM; 7 $\beta$-hydroxycholesterol, 7 $\beta$-OH;

20a-hydroxycholesterol, 20a-OH; 25-hydroxycholesterol, 25-OH; cholestane-3 $\beta, 5 a, 6 \beta$-triol, triol; cholesterol-5a,6a-epoxide, a-epoxide; 7-ketocholesterol, 7-keto

products increased significantly upon reheating after 3 storage days. However, $20 \alpha-\mathrm{OH}$ was not found in any of the samples reheated by different methods, and 7-keto was detected only in pan roasted luncheon meat reheated by microwave. $7 \beta-\mathrm{OH}, 25-\mathrm{OH}$, and $\alpha$-epoxide were present in greater amounts than other COPs and were found namely in sausages, loin ham, and bacon. $7 \beta-\mathrm{OH}$ was detected in all samples of sausages, loin ham, and bacon reheated after 3 days by different methods. Similarly, 25- $\mathrm{OH}$ and $\alpha$-epoxide were present in all reheated samples of loin ham. Pressed ham samples also showed cholesterol oxides after 3 days of storage, whereas no oxides were detected after cooking. $7 \beta-\mathrm{OH}$ was found to be present in the highest quantity $(497 \mu \mathrm{g} / 100 \mathrm{~g})$ pressed ham cooked by oven grilling and reheated with the same method after 3 days of storage. The ratio total amount of COPs/cholesterol in meat products increased relatively after 3 days of storage (0-0.86\%). The data presented in Table 4 shows that the highest ratio $(0.86 \%)$ was observed in cooked sausage reheated by microwave.

Generally, the amount of COPs in samples reheated after 6 days of storage at $4{ }^{\circ} \mathrm{C}$ (Table 5) was higher than 
Table 5 The amount of cholesterol oxidation products $(\mu \mathrm{g} / 100 \mathrm{~g})$ of meat products cooked with various methods and re-heated by microwaving at day 6 of storage at $4^{\circ} \mathrm{C}$

\begin{tabular}{|c|c|c|c|c|c|c|c|c|}
\hline \multirow[t]{2}{*}{ Sample } & \multirow{2}{*}{$\begin{array}{l}\text { Cooking } \\
\text { method }\end{array}$} & \multicolumn{6}{|c|}{ Cholesterol Oxidation Products } & \multirow{2}{*}{$\begin{array}{l}\text { COPs/cholestero } \\
(\%)\end{array}$} \\
\hline & & $7 \beta-\mathrm{OH}$ & $20 \mathrm{a}-\mathrm{OH}$ & $25-\mathrm{OH}$ & Triol & a-epoxide & 7-keto & \\
\hline \multirow[t]{6}{*}{ Sausage } & Fresh & $35.3 \pm 0.49$ & n.d. & $13.3 \pm 14.56$ & $41.8 \pm 68.60$ & $35.1 \pm 47.79$ & n.d. & $0.13 \pm 0.02^{C}$ \\
\hline & PP & $127.3 \pm 141.26$ & n.d. & $56.6 \pm 97.99$ & $43.8 \pm 75.89$ & $20.2 \pm 34.94$ & n.d. & $0.24 \pm 0.12^{C}$ \\
\hline & PM & $51.1 \pm 88.42$ & n.d. & $303.9 \pm 526.40$ & $72.9 \pm 126.26$ & n.d. & n.d. & $0.39 \pm 0.50^{C}$ \\
\hline & $\mathrm{OO}$ & $68.0 \pm 117.73$ & n.d. & $36.9 \pm 63.91$ & $125.9 \pm 218.12$ & n.d. & n.d. & $0.25 \pm 0.15^{C}$ \\
\hline & OM & $194.3 \pm 242.03$ & n.d. & $29.9 \pm 51.78$ & $913.8 \pm 1582.68$ & $130.0 \pm 225.16$ & n.d. & $1.32 \pm 1.81^{B C}$ \\
\hline & $\mathrm{MM}$ & $339.5 \pm 437.60$ & n.d. & $52.0 \pm 89.99$ & $120.6 \pm 187.71$ & n.d. & n.d. & $0.54 \pm 0.26^{C}$ \\
\hline \multirow[t]{6}{*}{ Loin ham } & Fresh & n.d. & n.d. & $43.7 \pm 75.79$ & n.d. & $25.8 \pm 44.74$ & n.d. & $0.07 \pm 0.13^{C}$ \\
\hline & PP & $14.1 \pm 24.33$ & n.d. & $147.2 \pm 232.12$ & $36.0 \pm 62.31$ & $119.7 \pm 167.93$ & n.d. & $0.57 \pm 0.70^{C}$ \\
\hline & PM & $9.3 \pm 16.11$ & n.d. & n.d. & n.d. & $36.7 \pm 63.53$ & n.d. & $0.09 \pm 0.11^{c}$ \\
\hline & $\mathrm{OO}$ & $15.6 \pm 26.95$ & n.d. & $114.9 \pm 156.50$ & $79.7 \pm 138.07$ & $191.0 \pm 204.85$ & n.d. & $0.39 \pm 0.30^{C}$ \\
\hline & OM & $17.2 \pm 29.86$ & n.d. & $243.7 \pm 388.99$ & $125.2 \pm 216.89$ & $188.8 \pm 178.94$ & $37.7 \pm 65.30$ & $0.59 \pm 0.48^{C}$ \\
\hline & MM & $23.5 \pm 40.75$ & n.d. & $131.33 \pm 187.97$ & - & $50.8 \pm 87.99$ & n.d. & $0.45 \pm 0.44^{c}$ \\
\hline \multirow[t]{6}{*}{ Bacon } & Fresh & n.d. & n.d. & n.d. & n.d. & n.d. & n.d. & $0.00 \pm 0.00^{C}$ \\
\hline & PP & $141.4 \pm 244.96$ & n.d. & n.d. & $164.2 \pm 284.39$ & n.d. & n.d. & $0.19 \pm 0.17^{c}$ \\
\hline & PM & $98.1 \pm 069.85$ & n.d. & n.d. & $106.9 \pm 105.64$ & $238.0 \pm 412.15$ & n.d. & $0.28 \pm 0.30^{C}$ \\
\hline & $\mathrm{OO}$ & $98.7 \pm 100.05$ & n.d. & n.d. & $228.2 \pm 395.25$ & n.d. & n.d. & $0.25 \pm 0.24^{C}$ \\
\hline & $\mathrm{OM}$ & $75.9 \pm 131.39$ & n.d. & $1718.6 \pm 2976.73$ & $2646.3 \pm 4583.49$ & $358.7 \pm 470.27$ & n.d. & $3.30 \pm 2.29^{\mathrm{A}}$ \\
\hline & MM & $91.1 \pm 157.75$ & $52.8 \pm 91.46$ & n.d. & n.d. & $347.6 \pm 602.05$ & n.d. & $0.32 \pm 0.31^{c}$ \\
\hline \multirow[t]{6}{*}{ Luncheon meat } & Fresh & n.d. & n.d. & n.d. & $154.4 \pm 267.47$ & $69.2 \pm 77.20$ & n.d. & $0.22 \pm 0.26^{C}$ \\
\hline & PP & n.d. & n.d. & n.d. & n.d. & n.d. & n.d. & $0.00 \pm 0.00^{C}$ \\
\hline & PM & $122.8 \pm 212.66$ & $47.9 \pm 82.89$ & n.d. & n.d. & n.d. & n.d. & $0.21 \pm 0.36^{C}$ \\
\hline & $\mathrm{OO}$ & n.d. & n.d. & $82.1 \pm 142.08$ & $158.8 \pm 274.96$ & n.d. & n.d. & $0.26 \pm 0.44^{C}$ \\
\hline & $\mathrm{OM}$ & $888.6 \pm 1539.04$ & $22.4 \pm 38.75$ & $84.4 \pm 146.13$ & n.d. & $102.0 \pm 176.73$ & n.d. & $1.20 \pm 1.56^{B C}$ \\
\hline & MM & $142.2 \pm 246.24$ & $27.2 \pm 47.47$ & n.d. & n.d. & n.d. & n.d. & $0.23 \pm 0.40$ \\
\hline \multirow[t]{6}{*}{ Press ham } & Fresh & n.d. & n.d. & $48.1 \pm 76.50$ & n.d. & $73.9 \pm 111.53$ & n.d. & $0.16 \pm 0.11$ \\
\hline & PP & n.d. & $19.7 \pm 34.07$ & $96.0 \pm 138.77$ & n.d. & $26.4 \pm 45.66$ & $18.3 \pm 31.77$ & $0.20 \pm 0.18$ \\
\hline & PM & n.d. & n.d. & n.d. & $449.8 \pm 779.10$ & $164.8 \pm 285.46$ & n.d. & $0.81 \pm 1.40^{C}$ \\
\hline & $\mathrm{OO}$ & $1017.2 \pm 1761.78$ & n.d. & n.d. & n.d. & n.d. & n.d. & $1.63 \pm 2.83^{\mathrm{ABC}}$ \\
\hline & $\mathrm{OM}$ & $1175.2 \pm 2012.57$ & n.d. & n.d. & $461.2 \pm 798.81$ & $164.6 \pm 285.09$ & n.d. & $2.87 \pm 2.78^{\mathrm{AB}}$ \\
\hline & MM & n.d. & $37.7 \pm 65.31$ & n.d. & n.d. & n.d. & $177.7 \pm 307.76$ & $0.36 \pm 0.46^{C}$ \\
\hline
\end{tabular}

Means \pm SE with different superscript in the same column ${ }^{A-C}$ ) differ significantly

Pan roasting (cooking) + pan roasting (re-heating), PP; pan roasting (cooking) + microwaving (re-heating), PM; oven grilling (cooking) + oven grilling (re-heating), OO; oven grilling (cooking) + microwaving (re-heating), OM; microwaving (cooking) + microwaving (re-heating), MM; 7 $\beta$-hydroxycholesterol, 7 $\beta$-OH;

20a-hydroxycholesterol, 20a-OH; 25-hydroxycholesterol, 25-OH; cholestane-3 $\beta, 5 a, 6 \beta$-triol, triol; cholesterol-5a,6a-epoxide, a-epoxide; 7-ketocholesterol, 7-keto

that in samples reheated after 3 days of storage, suggesting that increasing the storage time after cooking increased the COP content in processed meat products. $20 \alpha-\mathrm{OH}$ was not detected in any of the cooked samples nor in those reheated after 3 days; however, it appeared in samples reheated after 6 days of storage. A similar trend was also observed for 7-keto. The ratio between the total amount of COPs and cholesterol in meat products also increased after 6 days of storage (0.00-3.30\%), with the highest ratio in bacon cooked by oven grilling and reheated by microwave. The concentration of COPs in pressed ham was not detected after cooking and increased significantly upon reheating; the highest total amount of COPs/cholesterol ratios were observed in OM $(2.87 \%)$ and OO (1.63 \%). Our findings are in line with previous investigations, indicating that storage of cooked meat results in an increase in COP concentration upon reheating. Monahan et al. [28] reported that the rate of cholesterol oxidation in pork products significantly increased during storage after cooking. A higher level of lipid oxidation was found by El-Alim et al. [29] in microwave cooked meat patties after 7 storage days at $4{ }^{\circ} \mathrm{C}$. $\mathrm{Hu}$ 
et al. [15] also observed significantly higher COP levels in microwave cooked samples compared with the COP content in samples cooked by other methods. Processed meat initially free of COPs showed a minor increase in COP content during storage at $-4{ }^{\circ} \mathrm{C}$ for 4 weeks and a reduction of 15 to $24 \%$ in free cholesterol [30]. Juarez et al. [31] investigated the impact of different cooking methods on buffalo meat composition. Greater oxidative changes were observed in boiled and grilled samples, based on higher TBARS (thiobarbituric acid reactive substances) values. Ferioli et al. [32] evaluated lipid and cholesterol oxidation in fresh and cooked minced beef stored at $4{ }^{\circ} \mathrm{C}$; the COP content increased by 6 times after 2 weeks of storage. They also revealed that the increase in the COP content was higher in cooked meat than in fresh samples. Our investigation confirms these results, as a significant increase in the COP content was detected after refrigerated storage of the cooked meat samples.

\section{Conclusions}

Cooking of processed meat products resulted in a decrease of lipids and total cholesterol and in an increase in COP formation. Microwaving and oven grilling led to the production of higher amounts of COPs as compared with other cooking methods. The refrigerated storage of cooked products and subsequent reheating remarkably increased the amount of COPs in processed meat products. However, this increase was found to be lower in fresh products than in cooked products.

\footnotetext{
Abbreviations

COPs: Cholesterol oxidation products; BHT: Butylated hydroxyl toluene; GC: Gas chromatography; SAS: Statistical analysis system; 7 $\beta-\mathrm{OH}: 7 \beta-$ hydroxycholesterol; 20a-OH: 7a-hydroxy-cholesterol; 25-OH: 25hydroxycholesterol; Triol: Cholestanetriol; a-epoxide: 5,6a-epoxycholesterol; 7-keto: 7-ketocholesterol.
}

\section{Competing interests}

The authors declare that they have no competing interests.

\section{Authors' contributions}

The contribution of each authors to have this manuscript are as follow: MIK analyzed the data and proofread the manuscript to its final shape. JSM and SOL have planed the study design, conducted study analyses and prepared preliminary manuscript. DGY and KHS conducted the analysis of samples for COPs. ML assisted in statistical analysis of data and experimental designing and funding. CJ provide technical assistance during experiment, provide funding for experiments and help in manuscript preparation. It is confirmed that all authors have read the manuscript and approve its final version for submission.

\section{Acknowledgements}

This work was carried out with the support of Cooperative Research Program for Agriculture Science \& Technology Development (Project No. 011617), Rural Development Administration and Research Institute of Eco-friendly Livestock Science, Institute of Green Bio Science and Technology, Seoul National University, Korea.

\section{Author details}

${ }^{1}$ Department of Agricultural Biotechnology, Center for Food and Bioconvergence, and Research Institute of Agriculture and Life Science, Seoul National University, Seoul 151-921, South Korea. ${ }^{2}$ National Institute of Food
Science and Technology, University of Agriculture, Faisalabad 38040, Pakistan. ${ }^{3} \mathrm{CJ}$ Food Research Center, Seoul 152-050, Korea. ${ }^{4}$ Department of Health Administration and Food Hygiene, Jinju Health College, Jinju 660-757, South Korea. ${ }^{5}$ National Institute of Animal Science, RDA, Cheonan 331-801, South Korea. ${ }^{6}$ College of Agriculture and Life Science, Arsi University, Asella, Ethiopia.

Received: 30 June 2015 Accepted: 3 August 2015

Published online: 11 August 2015

\section{References}

1. Conchillo A, Ansoreno D, Astiasaran I. Intensity of lipid oxidation and formation of cholesterol oxidation products during frozen storage of raw and cooked chicken. J Sci Food Agric. 2005;85:141-6.

2. Rodriguez-Estrada MT, Penazzi G, Caboni MF, Bertacco G, Lercker G. Effect of different cooking methods on some lipid and protein components of hamburgers. Meat Sci. 1997:45(3):365-75.

3. Bou R, Codony R, Tres A, Decker EA, Guardiola F. Dietary strategies to improve nutritional value, oxidative stability, and sensory properties of poultry products. Crit Rev Food Sci Nutr. 2009;49:800-22.

4. Luna A, Labaque MC, Zygadlo JA, Marin RH. Effects of thymol and carvacrol feed supplementation on lipid oxidation in broiler meat. Poult Sci. 2010;8:366-70

5. Yin $\mathrm{H}$, Porter NA. New insights regarding the autoxidation of polyunsaturated fatty acids. Antiox Red Sig. 2005;7(1-2):170-84.

6. Linseisen J, Wolfram G. Origin, metabolism and adverse health effects of cholesterol oxidation products. Fett-Lipid. 1998;100:211-8.

7. Chizzolini R, Zanardi E, Dorigoni V, Ghidini S. Calorific value and cholesterol content of normal and low-fat meat and meat products. Trends Food Sci Tech. 1999:10:119-28.

8. Ryan E, Chopra J, McCarthy F, Maguire AR, O'Brien NM. Qualitative and quantitative comparison of the cytotoxic and apoptotic potential of phytosterol oxidation products with their corresponding cholesterol oxidation products. Brit J Nutr. 2005;94:443-51.

9. Garcla-Cruset S, Carpenter KLH, Codony R, Guardiola F. Cholesterol oxidation products and atherosclerosis. In: Cholesterol and Phytosterol Oxidation Products. Analysis, Occurrence and Biological Effects. Champaign, IL: AOCS Press; 2002.

10. Lee SO, Lim DG, Seol KH, Erwanto Y, Lee M. Effects of various cooking and re-heating methods on cholesterol oxidation products of beef loin. Asian Australas J Anim Sci. 2006;19:756-62.

11. McCluskey S. Cholesterol oxidation products in whole milk powders. Ph.D. Thesis. Dublin: Dublin City University; 1997.

12. Lee Jl, Kang S, Ahn DU, Lee M. Formation of cholesterol oxides in irradiated raw and cooked chicken meat during storage. Poult Sci. 2001;80:105-8.

13. Lillienberg $L$, Svanborg V. Determination of plasma cholesterol: comparison of gas liquid chromatography, colorimetric and enzymatic analysis. Clin Chim Acta. 1976:68:223.

14. Dominguez R, Gomez M, Fonseca S, Lorenzo JM. Effect of different cooking methods on lipid oxidation and formation of volatile compounds in foal meat. Meat Sci. 2014:97:223-30.

15. Hu SJ, Lee SY, Moon SS, Lee SJ. In Vitro effect of cooking methods on digestibility of lipids and formation of cholesterol oxidation products in pork. Korean J Food Sci An. 2014;34(3):280-6.

16. Freitas MT, Amaral CAA, Coutrim MX, Afonso RJCF, Junqueira RG. Effect of cooking method on the formation of 7-ketocholesterol in Atlantic hake (Merluccius hubbsi) and smooth weakfish (Cynoscion leiarchus) fillets. LWT-Food Sci Technol. 2015;62(2):1141-7.

17. Lee M, Sebranek JG, Olson DG, Dickson JS. Irradiation and packaging of fresh meat and poultry. J Food Prot. 1996;59:62-72.

18. Park SW, Addis PB. HPLC determination of C-7 oxidized cholesterol derivatives in foods. J Food Sci. 1985:50:1437-41.

19. Zubillaga MP, Maerker G. Quantification of three cholesterol oxidation products in raw meat and chicken. J Food Sci. 1991;56:1194-6.

20. Folch J, Lees M, Sloan-Stanley GH. A simple method for the isolation and purification of total lipids from animal tissues. J Biol Chem. 1957;226:497-509.

21. Badiani A, Stipa S, Bitossi F, Gatta PP, Vignola G, Chizzolini R. Lipid composition, retention and oxidation in fresh and completely trimmed beef muscles as affected by common culinary practices. Meat Sci. 2002;60:169-86. 
22. Baggio SR, Bragagnolo N. Cholesterol oxide, cholesterol, total lipid and fatty acid contents in processed meat products during storage. LWT-Food Sci Technol. 2006;39(5):513-20.

23. Du M, Ahn DU, Mendonca AF, Wesley IV. Quality characteristics of irradiated reday-to-eat turkey breast rolls from turkeys fed conjugated linoleic acid. Poult Sci. 2002:81(9):1378-84.

24. Broncano JM, Petron MJ, Parra V, Timon ML. Effect of different cooking methods on lipid oxidation and formation of free cholesterol oxidation products (COPs) in Latissimus dorsi muscle of Iberian pigs. Meat Sci. 2009;83:431-7.

25. Grau A, Codony R, Grimpa S, Baucells MD, Guardiola F. Cholesterol oxidation in frozen dark chicken meat: influence of dietary fat sources, and atocopherol and ascorbic acid supplementation. Meat Sci. 2001;57:197-208.

26. Ghiretti GP, Zanardi E, Novelli E, Campanini G, Dazzi G, Madarena G, et al. Comparative evaluation of some antioxidants in salame Milano and Mortadella production. Meat Sci. 1997;47:167-76.

27. Serra A, Conte G, Cappucci A, Casarosa L, Melle M. Cholesterol and fatty acids oxidation in meat from three muscles of Massese suckling lambs slaughtered at different weights. Italian J Anim Sci. 2014;13(3):648-52.

28. Monahan FJ, Gray Jl, Boreen AM, Miller ER, Buckley DJ, Morrissey PA, et al. Influence of dietary treatment on lipid and cholesterol oxidation in pork. J Agric Food Chem. 1992;40:1310-5.

29. El-Alim SSL, Lugasi A, Hovari J, Dworschak E. Culinary herbs inhibit lipid oxidation in raw and cooked minced meat patties during storage. J Sci Food Agric. 1999;79:277-85.

30. Vicente SJV, Torres EAFS. Formation of four cholesterol oxidation products and loss of free lipids, cholesterol and water in beef hamburgers as a function of thermal processing. Food Control. 2007:18:63-8.

31. Juarez J, Failla S, Ficco A, Pena F, Aviles C, Polvillo O. Buffalo meat composition as affect by different cooking methods. Food Bioprod Process. 2010;88:145-8.

32. Ferioli F, Caboni MF, Dutta PC. Evaluation of cholesterol and lipid oxidation in raw and cooked minced beef stored under oxygen enriched atmosphere. Meat Sci. 2008;80:681-5.

\section{Submit your next manuscript to BioMed Central and take full advantage of:}

- Convenient online submission

- Thorough peer review

- No space constraints or color figure charges

- Immediate publication on acceptance

- Inclusion in PubMed, CAS, Scopus and Google Scholar

- Research which is freely available for redistribution 